\title{
Video Article \\ A Novel and Translational Rat Model of Concussion Combining Force and Rotation with In Vivo Cerebral Microdialysis
}

\author{
Ian O. Massé ${ }^{1}$, Luc Moquin ${ }^{2}$, Chloé Provost ${ }^{1}$, Samuel Guay ${ }^{1}$, Alain Gratton ${ }^{2}$, Louis De Beaumont ${ }^{1}$ \\ ${ }^{1}$ Research Center, Hôpital du Sacré-Cœur de Montréal \\ ${ }^{2}$ Research Center, Douglas Institute
}

Correspondence to: Ian O. Massé at ian.masse.im@gmail.com

URL: https://www.jove.com/video/59585

DOI: doi: $10.3791 / 59585$

Keywords: Neuroscience, Issue 149, mild traumatic brain injury, concussion, head acceleration, in vivo, cerebral microdialysis, rat

Date Published: 7/12/2019

Citation: Massé, I.O., Moquin, L., Provost, C., Guay, S., Gratton, A., De Beaumont, L. A Novel and Translational Rat Model of Concussion Combining Force and Rotation with In Vivo Cerebral Microdialysis. J. Vis. Exp. (149), e59585, doi:10.3791/59585 (2019).

\section{Abstract}

Persistent cognitive and motor symptoms are known consequences of concussions/mild traumatic brain injury (mTBls) that can be partly attributable to altered neurotransmission. Indeed, cerebral microdialysis studies in rodents have demonstrated an excessive extracellular glutamate release in the hippocampus within the first $10 \mathrm{~min}$ following trauma. Microdialysis offers the clear advantage of in vivo neurotransmitter continuous sampling while not having to sacrifice the animal. In addition to the aforementioned technique, a closed head injury model that exerts rapid acceleration and deceleration of the head and torso is needed, as such a factor is not available in many other animal models. The Wayne State weight-drop model mimics this essential component of human craniocerebral trauma, allowing the induction of an impact on the head of an unrestrained rodent with a falling weight. Our novel and translational rat model combines cerebral microdialysis with the Wayne State weight-drop model to study, in lightly anesthetized and unrestrained adult rats, the acute changes in extracellular neurotransmitter levels following concussion. In this protocol, the microdialysis probe was inserted inside the hippocampus as region of interest, and was left inserted in the brain at impact. There is a high density of terminals and receptors in the hippocampus, making it a relevant region to document altered neurotransmission following concussion. When applied to adult Sprague-Dawley rats, our combined model induced increases in hippocampal extracellular glutamate concentrations within the first $10 \mathrm{~min}$, consistent with the previously reported post-concussion symptomology. This combined weight-drop model provides a reliable tool for researchers to study early therapeutic responses to concussions in addition to repetitive brain injury, since this protocol induces a closed-head mild trauma.

\section{Video Link}

The video component of this article can be found at https://www.jove.com/video/59585/

\section{Introduction}

The purpose of this method is to provide researchers with a reliable tool that faithfully reproduces the biomechanics of human craniocerebral trauma while allowing longitudinal characterization of the molecular effects of concussions/mild traumatic brain injury (mTBIs). This method combines cerebral microdialysis with the Wayne State weight-drop model to document, in lightly anesthetized and unrestrained adult rats, the acute changes in extracellular neurotransmitter levels following concussion. With this minimally-invasive method, neurotransmitters such as glutamate, GABA, taurine, glycine and serine can be rapidly and continuously quantified following trauma, in vivo, while not having to sacrifice the animal.

Concussion/mTBI is a pathophysiological disruption that affect brain functioning caused by an external force mechanism. Concussion/mTBI is the most common form of traumatic brain injury, accounting for $70-90 \%$ of cases ${ }^{1}$. Most of the acute functional disruptions following a concussion can be attributed to a primary and a secondary brain injury, ${ }^{2,3}$ (1) the primary brain injury is induced by the rapid acceleration and deceleration of the head and torso which damages brain tissues by compression followed by the stretching and shearing of axons during the backlash ${ }^{4,5,6}$ and (2) the secondary brain injury is the indirect cellular response to the trauma. It takes place hours and days after the primary brain injury and plays an important role in the motor and cognitive impairment observed over time. Many of the symptoms can be attributed to altered neurotransmission such as the previously demonstrated excessive extracellular glutamate release in the first 10 min following injury ${ }^{7,8,9}$. Given its high density of terminals and receptors, the hippocampus is a brain structure particularly vulnerable to this excitotoxic response following injury. Being heavily involved in cognitive function ${ }^{10,11}$, studies in rodents reported that hippocampal damage associated with concussion can lead to impairments in fear conditioning and learning spatial memory ${ }^{12,13}$. The primary objective of this methodology was to work out a rat model of concussion/mTBI, using the Wayne State closed head weight-drop procedure to faithfully reproduce the mechanisms of the primary brain injury, and incorporate cerebral microdialysis to study in vivo, the acute extracellular neurotransmitter changes due to the secondary brain injury following a concussion. Concentrations of extracellular glutamate and GABA were measured in the hippocampus to act as representative results of our method.

Previous rodent studies have combined microdialysis and other models of injury, such as the open-skull weight drop and controlled cortical impact, to demonstrate the acute changes in extracellular neurotransmitter levels following an injury of varying severity degrees ${ }^{14,15,16,17}$. 
However, in addition to the high degrees of variability, the translational value of models like the open-skull weight drop and controlled cortical impact is hampered by an inherent lack of ecological validity due to 2 factors: (1) these models induce injuries much more severe than sportrelated concussions suffered in humans, involving direct brain loading and (2) these models necessitate a craniectomy or a craniotomy, the head of the rodent being completely restrained in a stereotaxic frame, impeding the rapid acceleration and deceleration of the head and torso, thus poorly reproducing the biomechanics of concussion.

Microdialysis is a minimally-invasive method which offers the clear advantage of sampling neurotransmitters such as glutamate, GABA, taurine, glycine and serine, in vivo and continuously following trauma, while not having to sacrifice the animal. In addition to the advantages offered by microdialysis, the Wayne State University developed a closed-skull weight-drop model (as opposed to open-skull from other models), which allows the induction of a mTBI on a lightly anesthetized and unrestrained rodent, thus allowing the rapid acceleration and deceleration of the head and torso ${ }^{18}$. As mentioned previously, the acceleration and deceleration of the head and torso is a core biomechanical feature of sportrelated concussions seen in humans that previous rodent $\mathrm{mTBI}$ models have failed to address. The weight-drop procedure can be done very quickly and does not require any prior surgery or scalp incision. Following induction of the concussion, rodents recover the righting reflex almost spontaneously and do not experience paralysis, seizures or respiratory distress after a single impact. Intracranial bleedings and skull fractures are rare, and only minor deficits in motor coordination have been reported in rodents. This rat model is easy-to-use, inexpensive and facilitates the quantification of neurotransmitters released in the acute phase following a concussion without removing the microdialysis probe during the impact.

Our rat model combining microdialysis and concussion is appropriate for researchers seeking to characterize longitudinally the molecular effects of concussion and could be used in a wide variety of therapeutical studies. Indeed, despite several years of research and an overwhelming need, no drug to prevent the long-term effects of concussions has passed the clinical trial phase ${ }^{19}$. One of the potential reasons for these failures could be the use of animal models that do not faithfully reproduce the traumatic biomechanical forces of concussions as experienced by humans. The method presented here meets the definition of human concussions which specifies that the primary brain injury is induced by a blunt impact as well as rapid acceleration and deceleration of the head and torso ${ }^{2,3}$.

Furthermore, our combined model is appropriate for researchers studying the effects of repeated mild traumatic brain injury (rmTBI) since one of its key characteristics that sets it apart from other animal models of concussion is that it makes it possible to induce repeated, mild injuries to the same case ${ }^{18}$. In humans, rmTBI is associated with more severe post-traumatic symptoms, longer recovery times, and aggravated motor and cognitive impairments that tend to spread over time $\mathrm{e}^{20,21}$. Other relevant animal models have also made it possible to better understand the post-traumatic pathophysiology of $\mathrm{rmTB}^{22,23,24,25,26,27}$. Increased brain vulnerability has been demonstrated in rodents after a minimum of $5 \mathrm{mTB}$ at $24 \mathrm{~h}$ intervals. Neuroinflammation increases with the number of $\mathrm{mTBI}$ experienced and markers of neurodegeneration appear ${ }^{28}$. Repeated $\mathrm{mTBI}$ would prevent the transition of microglia from a proinflammatory mode to a normal mode of recovery, resulting in prolonged excitotoxic activity and activation of neurodegenerative mechanisms ${ }^{29}$. With our model, rats could be exposed to 1 impact per day over the period of 1 week for a total of 5 exposures. Given the simplicity of this animal model, it could facilitate the characterization of the cumulative effects of the acute indiscriminate neurotransmitter release arising immediately after a mTBI.

This model also allows animals to be readily exposed to 2 impacts per day, making it possible to study even more severe conditions such as when an athlete receives another traumatic impact within a short time from the first blow ${ }^{30}$. As demonstrated in a previous study ${ }^{31}$, the timing of a second blow to the head can dramatically affect vascular and axonal damage. The closer the second blow is to the first blow, the more damaging the consequences. This model is appropriate for investigating how this particular condition affects extracellular neurotransmitter release.

In this method, the hippocampus was used as region of interest due to its relevancy in concussion research but microdialysis samples can be collected from other regions of interest as well. However, any other brain region has to be considered on account of the space left by the impact site from the guide cannula, including the dental cement surrounding it, can take up a considerable amount of space on the rat's head. In addition to this, the microdialysis parameters presented in this method such as the membrane's molecular weight cut-off and active length, the sampling time intervals and the flow rate can be adjusted according to the type of molecule studied. The efficient collection of pro-inflammatory cytokines involved in concussions, for example, would require a membrane with a much larger pore size.

\section{Protocol}

The animal protocol for this project obtained the approval from the Animal Care Committee of the Hopital du Sacre-Cœur de Montreal in compliance with the guidelines of the Canadian Council on Animal Care.

NOTE: A schematic outline of the research protocol is presented in Figure 1.

\section{Animal Preparation}

1. Order Sprague-Dawley rats from a standard laboratory animal supplier to be delivered between 43 and 50 days of age and at a weight between 151 and $200 \mathrm{~g}$.

2. House all rats individually in a cycle of $12: 12 \mathrm{~h}$ light: darkness, at $24-26{ }^{\circ} \mathrm{C}$ with ad libitum access to water and food.

3. During the 2 weeks before starting the protocol, handle the rats for $5 \mathrm{~min}$ on a daily basis to facilitate their habituation in contact with researchers. Rats should be aged about 10 weeks old and their weight should be between 295 and $351 \mathrm{~g}$ at the time of concussion or sham injury induction.

\section{Microdialysis Guide Cannula Implantation Surgery}

1. Perform the surgery under sterile conditions. Wear sterile gloves, a hair bonnet and a surgical mask throughout the procedure. Autoclave and sterilize all the materials and surgical instruments beforehand. Clean and disinfect the working area and stereotaxic apparatus thoroughly with a solution of ethanol $(70 \%)$. 
2. Anesthetize the animals by injecting a cocktail of ketamine $(70 \mathrm{mg} / \mathrm{kg})$ and xylazine $(10 \mathrm{mg} / \mathrm{kg})$ intraperitoneally. Asses anesthetic depth by testing the reflex to a toe pinch.

3. Remove fur from the head of the animal using electric clippers. Clean shaved head using a solution of $2 \%$ isopropyl alcohol and $2 \%$ chlorhexidine gluconate (3 times). Apply lubricating eye ointment during the anesthesia to prevent dryness.

4. Drape-off the surgical field so that only the head of the animal is exposed. Place the head of the rat in a stereotaxic apparatus, insert the ear bars into the ear canals with great care then tighten the nose clamp. Fix a $26 \mathrm{G}$ stainless-steel guide cannula to the holder arm on the stereotaxic apparatus.

5. Locally inject an anesthetic cocktail of bupivacaine $(1.5 \mathrm{mg} / \mathrm{kg})$ and lidocaine $(1.5 \mathrm{mg} / \mathrm{kg})$ subcutaneously on the head, $10 \mathrm{~min}$ prior to incision.

6. Maintain anesthesia during the whole procedure by delivering sodium isoflurane $(2.5 \%)$ at $0.5 \mathrm{~L} / \mathrm{min}$ oxygen flow with a nose cone.

7. Make a midline incision $(3 \mathrm{~cm})$ along the scalp with a scalpel. Leave the skull clear by installing 4 clamps around the incision.

8. Scrape firmly the periosteum from the skull with a surgical blade until the Bregma and Lambda sutures are visible. Maintain firm pressure on the skull with a gauze pad or cotton tipped applicator if there is bleeding.

9. Confirm if the skull is correctly aligned on the stereotaxic apparatus by comparing the dorsoventral coordinates of the Bregma and Lambda sutures. Identify the anteroposterior, mediolateral and dorsoventral coordinates of the Bregma suture as the reference points for the coordinates of the guide cannula.

10. Taking the Bregma suture coordinates as references, calculate the coordinates of the guide cannula implantation site in the hippocampus. NOTE: The following coordinates were determined according to the rat brain atlas from Paxinos and Watson (anteroposterior: $-0.60 \mathrm{~cm}$; mediolateral: $\pm 0.58 \mathrm{~cm}$; dorsoventral: $-0.16 \mathrm{~cm}$, Figure $2 \mathrm{~A})^{32}$.

11. Mark the precise implantation site using a marker.

12. Drill a $0.5 \mathrm{~mm}$ hole through the cranium at the target site of the guide cannula. Drill 3 other holes approximately $5 \mathrm{~mm}$ around this point to thread 3 anchor screws into the skull that will solidify the cannula after acrylic dental cement is applied.

13. Insert the cannula into the hippocampus and fix it with dental cement. This cannula will be used to insert the probe into the region of interest 7 days later during the microdialysis procedure. Be careful as to not spill excess dental cement around the site where the weight will be dropped.

14. Leave the cement to dry for $2 \mathrm{~min}$, then remove the holder arm from the cannula. Insert a stainless-steel removeable obturator into the cannula to avoid cerebrospinal fluid seepage and risks of infection.

15. Remove the 4 clamps, pull back the retracted skin and stitch it with a surgical suture thread $4-0$.

16. Remove the rat from the apparatus and inject buprenorphine subcutaneously to treat pain $(0.05 \mathrm{mg} / \mathrm{kg}$, after surgery then once per day during the following 2 days). Place the rodent back in its cage with a heating pad under until it becomes conscious, then return it to the animal care facility for a 7 days recovery period under close monitoring.

\section{Microdialysis Procedure}

1. While performing the microdialysis procedure, wear sterile gloves, a hair bonnet and a surgical mask. Seven days following the cannula implantation surgery, anesthetize the rat with sodium isoflurane $(2.5 \%)$ at $0.5 \mathrm{~L} / \mathrm{min}$ oxygen flow.

2. Remove the obturator from the cannula and insert slowly a microdialysis probe, perfused with artificial cerebral spinal fluid (ACSF) (26 $\mathrm{mmol} / \mathrm{L} \mathrm{NaHCO} 3,3 \mathrm{mmol} / \mathrm{L} \mathrm{NaH} \mathrm{PO}_{4}, 1.3 \mathrm{mmol} / \mathrm{L} \mathrm{MgCl} 2,2.3 \mathrm{mmol} / \mathrm{L} \mathrm{CaCl} 2,3.0 \mathrm{mmol} / \mathrm{L} \mathrm{KCl}, 126 \mathrm{mmol} / \mathrm{L} \mathrm{NaCl}, 0.2 \mathrm{mmol} / \mathrm{L} \mathrm{L-ascorbic} \mathrm{acid),}$ through the cannula into the hippocampus or other region of interest.

NOTE: Rats need to be anesthetized only while removing the obturator and inserting the microdialysis probe, and during induction of concussion or sham injury. The probes used here are laboratory-constructed, l-shaped, and comprised of fused side-by-side silica inlet-outlet lines [internal diameter (ID): $50 \mu \mathrm{m}$ ] encased in polyethylene tubing (ID: $0.58-0.38 \mathrm{~mm}$ ). The end of the cannula is secured with a length of regenerated hollow cellulose membrane [molecular weight cut-off: $13 \mathrm{kDa}$, outer diameter (OD): $216 \mu \mathrm{m}$; ID: $200 \mu \mathrm{m}$ ] using cyanoacrylate adhesive and the tip sealed with epoxy. The active membrane measures $2.5 \mathrm{~mm}$ for implantation in the hippocampus but can be adjusted according to the depth of the region of interest. The connection of the indwelling cannula of the rat to the probe is secured with a fitted, threaded stainless-steel collar.

3. Fix the probe assembly to a stainless-steel spring tethered to a liquid swivel and counter balance lever arm suspended above the cage with a ring stand and clamps so that the animal can move freely within its cage. Tethered rats spend the entire duration of the microdialysis procedure with ad libitum access to water and food.

4. Use a microinfusion pump to send perfusate to the probes, and collect the dialysate from the fused silica outlet line (dead volume: $0.79 \mu \mathrm{L}$ ).

5. At least $1 \mathrm{~h}$ and 30 min before the procedure begins, turn up the probe to its working flow rate $(1 \mu \mathrm{L} / \mathrm{min})$. Verify that the flow rate of the probe is consistent by measuring volume over time with a pipette.

NOTE: The flow rate can be more or less depending on the neurotransmitters sampled and the brain region of interest. Dialysis samples are taken before, during, and after concussion or sham injury induction. Sampling interval depends on the brain region of interest, neurotransmitters being analyzed, dialysate concentrations of the analyte, and sensitivity of the analytical chemistry equipment used. The collecting phases done here in the hippocampus for glutamate and GABA sampling are as follows:

1. Baseline: At beginning of experiment, collect dialysis samples at $10 \mathrm{~min}$ intervals for $60 \mathrm{~min}$.

2. Post-concussion or sham injury: After concussion or sham injury, collect samples for an additional 90 min (9 samples).

6. Collect each dialysate sample in a fraction vial preloaded with $1 \mu \mathrm{L}$ of $0.25 \mathrm{~mol} / \mathrm{L}$ perchloric acid to prevent analyte degradation. Store the samples at $4{ }^{\circ} \mathrm{C}$ for subsequent analysis.

7. Following the collection of the last dialysate sample, re-anesthetize the rat with a nose cone delivering sodium isoflurane $(2.5 \%)$ at $0.5 \mathrm{~L} / \mathrm{min}$ oxygen flow.

8. Remove the microdialysis probe from the cannula, re-insert the obturator and then return the rat to the animal care facility. 


\section{Concussion Apparatus Installation}

1. Prior to commencement of procedure, carve a weight to be used to inflict the concussion (19 $\mathrm{mm}$ in diameter) from solid brass to obtain a mass of $450 \mathrm{~g}$. Insert a metal loop at the top of the brass weight. Drill holes preliminary at a distance of $1.0 \mathrm{~m}$ inside a vertical polyvinyl chloride (PVC) guide tube.

2. Slit an aluminum sheet with a sharp razor blade. The slotted aluminum sheet should support the weight of the rat (295 to $351 \mathrm{~g})$ without interfering with the acceleration of its body after head impact from the brass weight.

3. Tape the slotted aluminum sheet tightly to a U-shaped Plexiglas frame $(38 \mathrm{~cm}$ long $\times 27 \mathrm{~cm}$ wide $\times 30 \mathrm{~cm}$ deep, Figure $3 \mathbf{A}, \mathbf{B})$ that contains a foam cushion ( $37 \mathrm{~cm}$ long $\times 26 \mathrm{~cm}$ wide $\times 12 \mathrm{~cm}$ deep).

4. Position the Plexiglas frame under a PVC guide tube (20 mm diameter $\times 1.5 \mathrm{~m}$ length).

5. Hold the PVC guide tube in place with a clamp stand $3.5 \mathrm{~cm}$ above the slotted aluminum.

6. Attach a nylon fly fishing line (capacity of $9.1 \mathrm{~kg}, 0.46 \mathrm{~mm}$ diameter) through the metal loop so that the bottom of the weight hangs $2.5 \mathrm{~cm}$ over the slotted aluminum as to prevent multiple hits when the rat is falling on the foam cushion following impact.

7. Attach the nylon fly fishing line to the clamp stand.

8. Pull up the weight through the PVC tube with the nylon fly fishing line then keep it in place by inserting a hex key through the preliminary drilled holes at $1.0 \mathrm{~m}$.

\section{Concussion Induction}

1. After the baseline phase of the dialysis samples collection, re-anesthetize the rat lightly by placing a nose cone delivering sodium isoflurane ( $2.5 \%$ isoflurane at $0.5 \mathrm{~L} / \mathrm{min}$ oxygen flow) until it as no response to a toe pinch (as mentioned in section 3.1 ).

2. Place the animal on its chest on the slotted aluminum sheet so that its head is positioned directly in the path of the brass weight (Figure 3C,D). Maintain anesthesia with the nose cone to make sure that the rat does not move or wake-up before the weight strikes it.

3. Remove the nose cone and pull the hex key. The weight will fall vertically through the PVC tube and impact the head of the rat. The rat will undergo a rapid $180^{\circ}$ rotation and land on its back (Figure $3 E$ ).

4. Remove the rat from the foam cushion and place it on its back in its cage.

5. Use a digital timer to measure the righting reflex time as a sign of recovery and injury severity. The righting reflex time is the total time from the impact until the rodents wake up and spontaneously right themselves to the prone position from the supine position, or start walking. Note any signs of death, fracture, or bleeding.

NOTE: The procedure can be repeated on the same subject at different time points for repeated concussions.

\section{Sham Induction}

1. After the baseline phase of the dialysis samples collection, re-anesthetize the rat lightly by placing a nose cone delivering sodium isoflurane $(2.5 \%)$ at $0.5 \mathrm{~L} / \mathrm{min}$ oxygen flow until it as no response to a toe pinch (as mentioned in section 3.1).

2. Place the animal on its chest on the slotted aluminum sheet so that its head lays directly in the path of the brass weight. Maintain anesthesia with the nose cone to make sure the rat does not move or wake-up.

3. Remove the nose cone and remove the animal from the aluminum sheet without pulling the hex key. The rat will not undergo a rapid $180^{\circ}$ rotation.

4. Place the rat on its back in its cage.

5. Use a digital timer to measure the righting time as an indicator of neurologic restoration.

\section{High-performance Liquid Chromatography}

1. Determine neurotransmitter levels (i.e., glutamate and GABA) by precolumn derivatization using high-performance liquid chromatography with rapid separation fluorescence detection, and a system consisting of a rapid separation autosampler and a pump coupled to a $3.0 \times 50$ $\mathrm{mm} 5 \mu \mathrm{m}$ analytical column.

2. Prepare a mobile phase with $100 \mathrm{mmol} / \mathrm{L}$ sodium phosphate dibasic $\left(\mathrm{Na}_{2} \mathrm{HPO}_{4}\right), 3.5 \%$ acetonitrile and $20 \%$ methanol. Adjust the $\mathrm{pH}$ to 6.7 with phosphoric acid $(85 \%)$ as needed.

3. Set the flow rate to $0.5 \mathrm{~mL} / \mathrm{min}$.

4. Prepare fresh daily derivatization reagents and working standards $(100 \mathrm{ng} / \mathrm{mL})$ from stock solutions. Load them into a refrigerated $\left(10{ }^{\circ} \mathrm{C}\right)$ rapid separation autosampler with samples.

5. Mix each fraction sequentially into the analytical column with $20 \mu \mathrm{L}$ of 3-mercaptopropionic acid $(0.071 \mathrm{~mol} / \mathrm{L})$ diluted with $\mathrm{H}_{2} \mathrm{O}$ and $20 \mu \mathrm{L}$ of ophthaldehyde $(0.0143 \mathrm{~mol} / \mathrm{L})$ diluted with $0.1 \mathrm{~mol} / \mathrm{L}$ sodium tetraborate. Allow $10 \mathrm{~min}$ for the mix to react.

6. To prevent contamination of next samples, flush the injection loop with methanol (20\%), following each injection.

NOTE: The glutamate retention time would be of $1 \mathrm{~min}$ approximately in this protocol, for a total run time of 30 min for each sample.

7. During analysis of chromatographic peaks, identify unknown peaks using samples matched according to time of retention from known standards. Express levels of analytes as $\mu \mathrm{g} / \mathrm{mL}$.

\section{Histology}

1. A month following the microdialysis procedure and concussion or sham injury induction, anesthetize the animals by injecting a cocktail of ketamine $(70 \mathrm{mg} / \mathrm{kg})$ and xylazine $(10 \mathrm{mg} / \mathrm{kg})$ intraperitoneally and euthanize them by paraformaldehyde $(4 \%)$ and saline intracardiac perfusion.

2. Decapitate the rodents then dissect the brains.

3. Store the brains in paraformaldehyde $(4 \%)$ and subsequently cryoprotect them in a solution of sucrose $(30 \%)$. 
4. Slice the brains in coronal sections of $50 \mu \mathrm{m}$ with a cryostat.

5. Stain the brain slices with cresyl violet for histological verification of injury and probe placement (Nissl staining).

\section{Representative Results}

Using our model of concussion which combines force and rotation with in vivo cerebral microdialysis, the acute extracellular glutamate and GABA changes over time following a concussion or sham injury were investigated in 21 male, adult, Sprague-Dawley rats by the implantation of a guide cannula in the CA1 region of the hippocampus.

\section{Histological verification of probe placement and injury}

No morphological changes such as massive intracerebral hemorrhages or contusions were reported following the histological verification of hippocampus tissue damage on sections stained with cresyl violet. Guide cannula implantation and microdialysis probe insertion induced minor and similar damages between injured and sham cases. Moreover, not removing the probe right before sham injury or concussion induction did not yield any distinguishable hippocampus tissue damage as seen under a microscope (Figure 2B,C, respectively), with the membrane of the probe still intact afterwards (Figure 2D,E). Concussion and sham injury brains perfused with paraformaldehyde (4\%) 1 month following microdialysis procedures are indistinguishable upon visual inspection (Figure 2F,G).

\section{Righting reflex time}

Animals from the injured group had a significantly increased righting time on average versus sham cases (Student's t-test, $p=0.042801)$ (Figure 4) and appeared stunned upon regaining consciousness. Of the 10 cases from the concussion group, a single animal showed minor signs of bleeding under the impact site following the weight-drop. No other signs of skull fracture or intracranial bleeding were observed.

\section{In vivo cerebral microdialysis}

To act as representative results of our method, fifteen $10 \mu \mathrm{L}$ samples of dialysate were extracted from the hippocampus, in vivo, at intervals of 10 min and a flow rate of $1 \mu \mathrm{L} / \mathrm{min}$. Extracellular levels of glutamate and GABA were measured from 6 samples during baseline (60 min) and from 9 samples following induction of sham injury or concussion (90 min).

\section{Extracellular concentrations of glutamate}

Significant increases in extracellular glutamate concentrations were observed in the CA1 region of the hippocampus during the first 10 min following induction of trauma compared to sham injury (Mann-Whitney $U$ Test, $p=0.009175$ ) (Figure 5). No other difference in glutamate concentrations were observed between groups at any other time point.

\section{Extracellular concentrations of GABA}

No significant change in GABA concentrations were observed in the CA1 region of the hippocampus during the first 10 min following induction of trauma compared to sham injury (Mann-Whitney $U$ Test, $p=0.943861$ ) (Figure 6). There was no other significant difference in GABA concentrations at any other time point between concussion cases and sham injury cases.

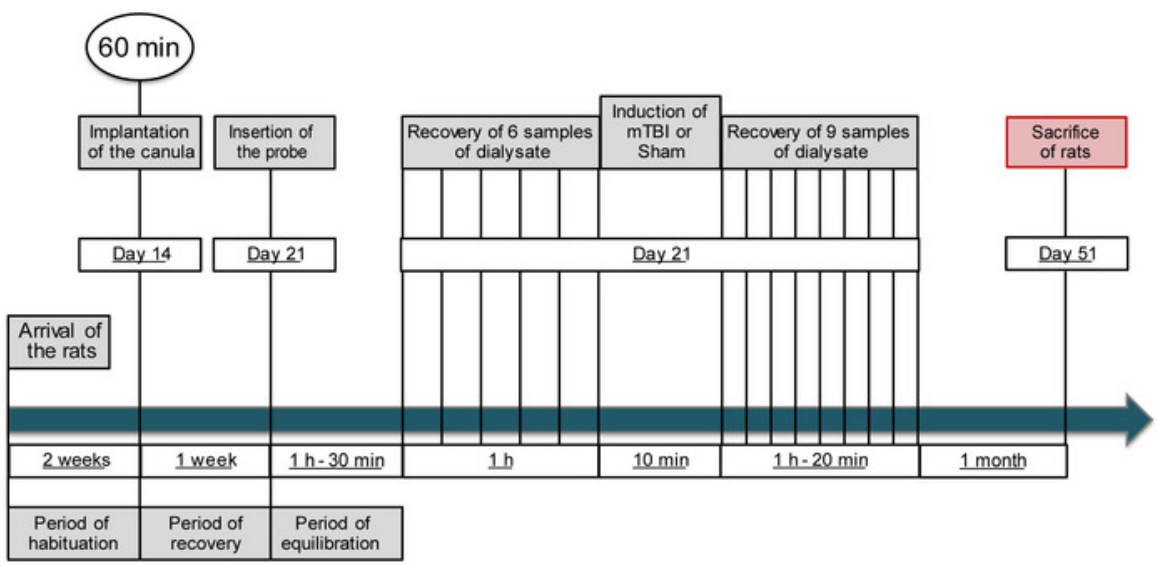

Figure 1: Schematic outline of the research protocol. This figure has been modified from IO Masse 2018. Please click here to view a larger version of this figure. 

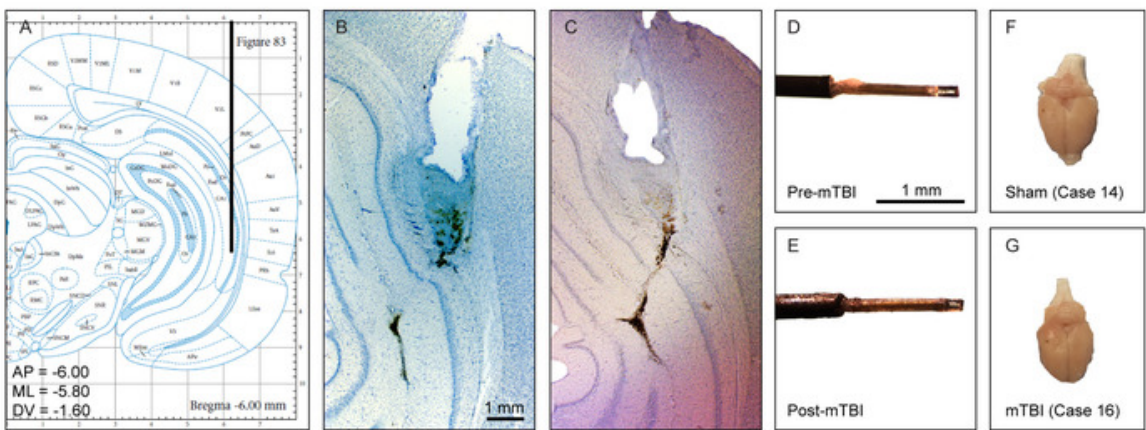

Figure 2: Histological verification of probe placement and injury. (A) Coronal view of the microdialysis probe and guide cannula placement site in the hippocampus using the stereotaxic atlas of Paxinos and Watson. (B) Representative photomicrograph of hippocampus tissue damage (cresyl violet) produced by a microdialysis probe and guide cannula from a sham injury case. (C) Representative photomicrograph of hippocampus tissue damage (cresyl violet) produced by a microdialysis probe and guide cannula from a concussion case. (D) Representative photomicrograph of a microdialysis probe before induction of concussion. (E) Representative photomicrograph of a microdialysis probe after induction of concussion. The membrane is still intact. (F-G). Representative photomicrograph of a sham (F) and concussion (G) injured brain following perfusion with $4 \%$ paraformaldehyde at 1-month after sham injury or concussion procedure. Upon visual inspection, the 2 brains are indistinguishable. This figure has been modified from IO Masse 2018. Please click here to view a larger version of this figure.
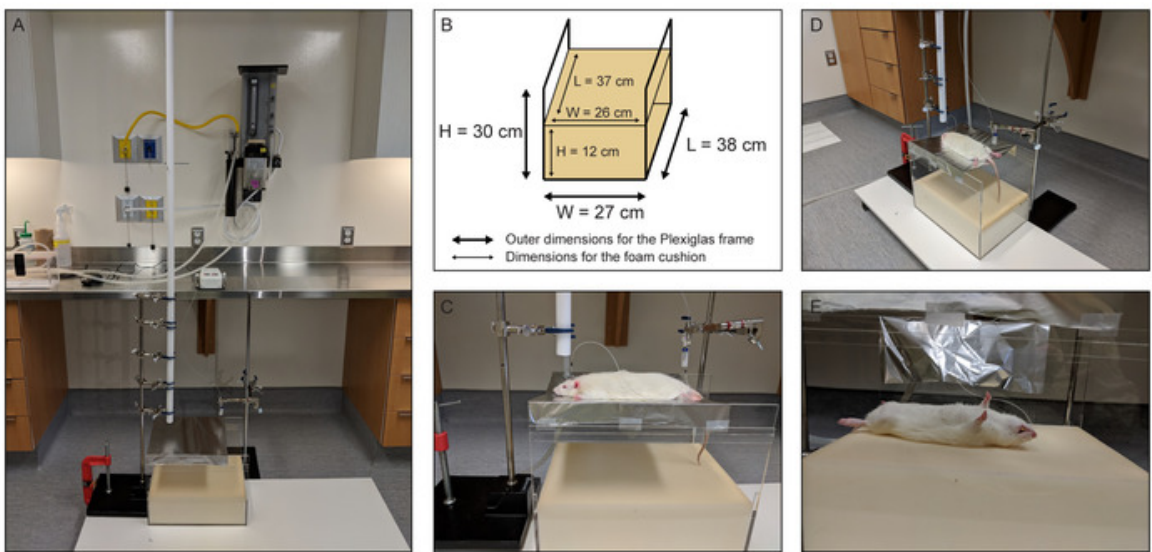

Figure 3: Concussion apparatus and microdialysis instruments essential components depictions. (A) A photograph of the entire assembly comprised of a vertical polyvinyl chloride (PVC) guide tube for the falling weight situated above the rat stage, Plexiglas frame, foam cushion, computer-controlled microinfusion pump, gastight syringes, liquid swivels, and side-by-side fused silica inlet-outlet lines. (B) Schematic representation of the Plexiglas frame and foam cushion with all pertinent dimensions. (C) A photograph of the slotted piece of aluminum foil that serves as the rat stage above the foam cushion. (D) A photograph showing the positioning of the rat on the stage immediately prior to head impact by the falling weight. (E) A photograph showing the rat after head impact, illustrating the $180^{\circ}$ horizontal rotation of the body of the rat after the head impact and ensuing acceleration and rotation. This figure has been modified from IO Masse 2018. Please click here to view a larger version of this figure. 


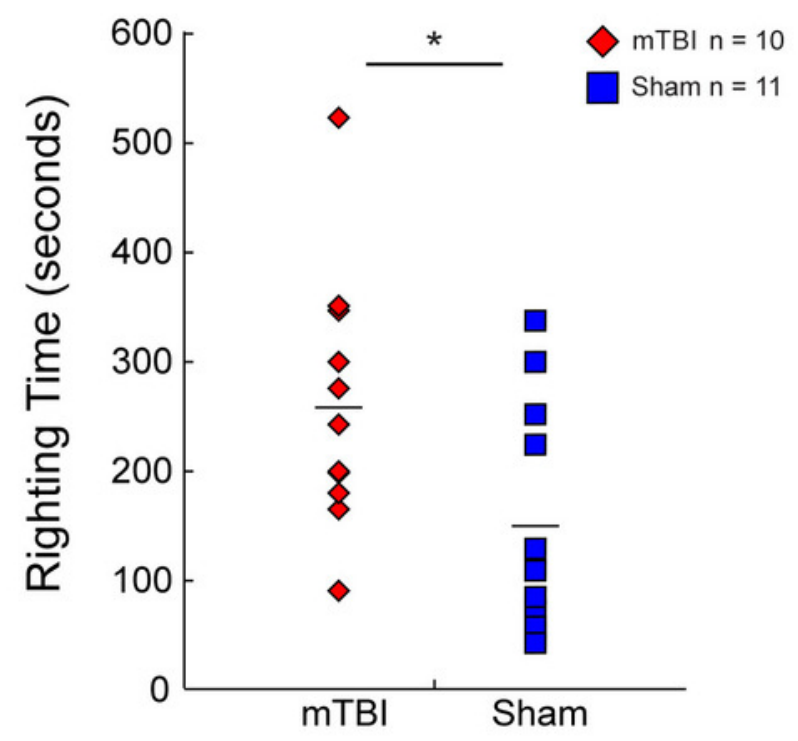

Figure 4: Righting time. Histogram representations of the time taken by rats to wake from the anesthetic and flip from the supine position to the prone position or begin walking following concussion (red diamonds, $n=10$ ) or sham injury (blue squares, $n=11$ ). Rats from the concussion group took significantly longer to right themselves compared to the sham injury group. Mean values are represented as a horizontal line in each graph. ${ }^{*} p<0.05,{ }^{* *} p<0.01,{ }^{* * *} p<0.001$. This figure has been modified from IO Masse 2018. Please click here to view a larger version of this figure.

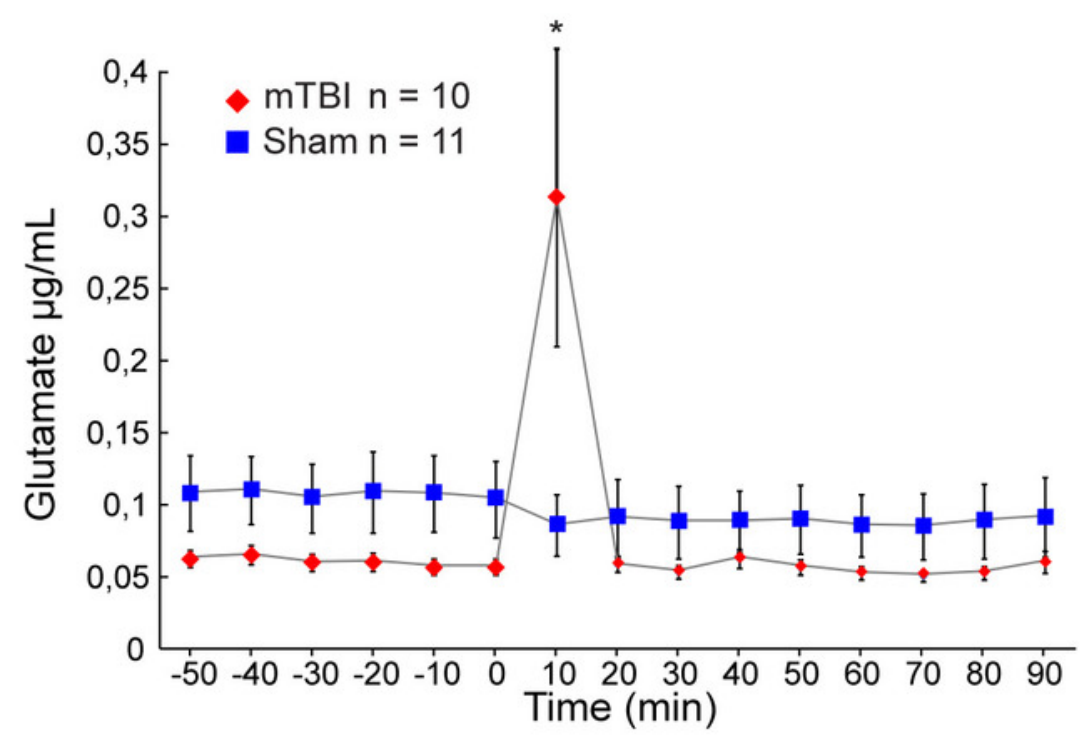

Figure 5: Extracellular concentrations of glutamate. Mean extracellular concentrations of glutamate $(\mu \mathrm{g} / \mathrm{mL}) \mathrm{measured} \mathrm{by} \mathrm{microdialysis} \mathrm{in}$ the hippocampus during baseline $(60 \mathrm{~min}$ ) and after concussion (red diamonds, $n=10$ ) or sham injury (blue squares, $n=11)$ conditions $(90$ $\mathrm{min}$ ). Error bars represent the standard error of mean. ${ }^{*} \mathrm{P}<0.05$, ${ }^{* *} \mathrm{P}<0.01$, ${ }^{* \star *} \mathrm{P}<0.001$. This figure has been modified from IO Masse 2018 . Please click here to view a larger version of this figure. 


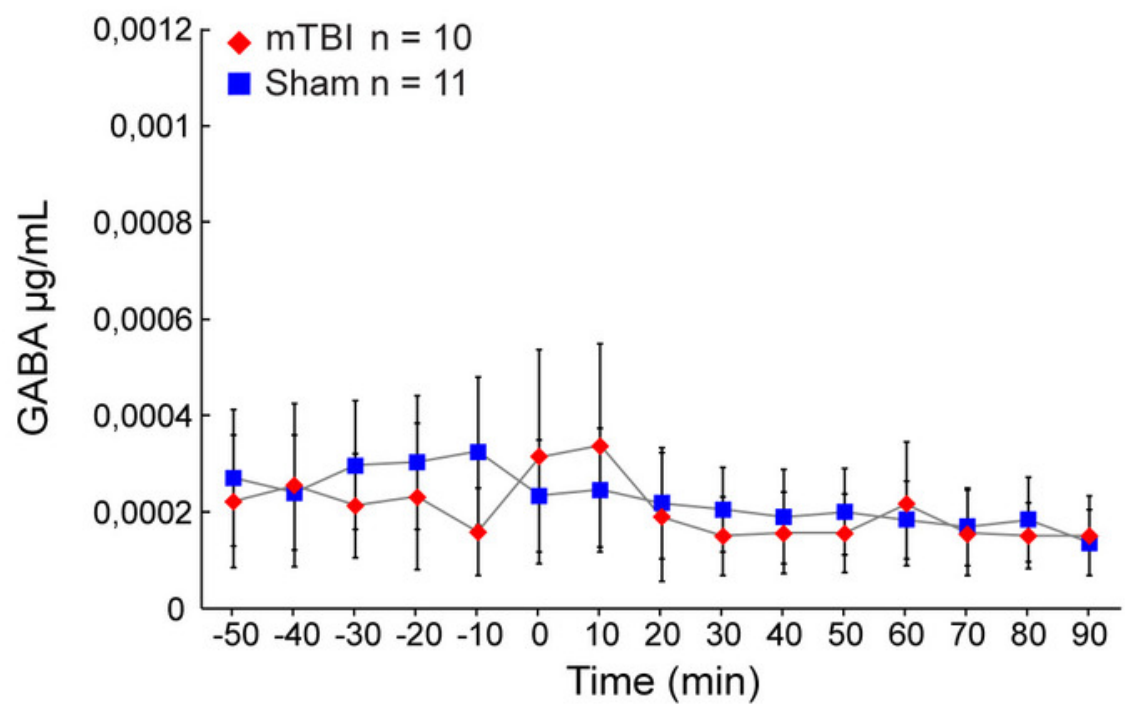

Figure 6: Extracellular concentrations of GABA. Mean extracellular concentrations of GABA $(\mu \mathrm{g} / \mathrm{mL})$ measured by microdialysis in the hippocampus during baseline (60 $\mathrm{min}$ ) and after concussion (red diamonds, $n=10)$ or sham injury (blue squares, $n=11)$ conditions $(90$ min). Error bars represent the standard error of mean. ${ }^{*} \mathrm{P}<0.05$, ${ }^{* *} \mathrm{P}<0.01$, ${ }^{* * *} \mathrm{P}<0.001$. This figure has been modified from IO Masse 2018 . Please click here to view a larger version of this figure.

\section{Discussion}

\section{Critical steps in the protocol}

For the generation of reliable results, critical steps in this protocol require particular attention. During the cannula implantation surgery, avoid using more cement than necessary, especially when it is very liquid as to prevent spilling over the impact site. To avoid blocking the implantation site, use an obturator that is the same length as the cannula. During the microdialysis procedure, insert the probe slowly into the cannula and make sure that it is inserted completely for dialysate sampling. Before the concussion induction, make sure that the aluminum sheet is properly slotted with a sharp razor blade. Otherwise, the impact from the brass weight won't be sufficient to rip the aluminum sheet and the rat will remain chest down instead of undergoing an $180^{\circ}$ rotation and landing on its back. If this is the case, injuries induced will result from the blunt impact, not unlike what is seen in the open-skull weight drop models and be significantly more severe. During the concussion induction, avoid impacting the cannula with the weight as this would generate critical damage to the skull of the rat. It is highly recommended to work in teams of 2 to restrict manipulation errors during the experiment.

\section{Modifications and troubleshooting}

During the microdialysis procedure, flow should be constant and yield a volume appropriate to the rate of perfusion, once the probe is linked to the pump. Lower volumes can indicate the presence of clogging in the membrane of the probe or air bubbles in the lines. In the case of clogging, the probe should be discarded and replaced. However, air bubbles can be ejected by circulating ACSF in the lines. If there is no clogging or air bubbles noted and there is still no flow, a small part of the outflow tube nearest to the end can be cut.

\section{Limitations of the method}

Other studies using the Wayne State University weight-drop have evaluated some fundamental structural and molecular changes ${ }^{18}$. However, a more extensive investigation would maintain the legitimacy of this procedure. Information regarding the biological and neuroanatomical changes that take place at epigenetic and cellular levels would further solidify the reliable and translational value of our method. Furthermore, evaluation of cognitive function is a reliable measure of outcome related to $\mathrm{mTBI}$ in rodent models ${ }^{33}$. While the time-to-right was measured in this protocol and was significantly delayed in injured cases compared to sham cases, studies in the future should concentrate on methodically measuring cognitive function following trauma induction in rodents.

\section{Significance of the method with respect to existing/alternative methods.}

The main significance of the method is twofold: Firstly, it allows the successful induction of a concussion with the Wayne State University procedure, which allows rapid acceleration and deceleration of the head and torso. With this method, serious injury outcomes such as cardiorespiratory arrests, skull fracture, high mortality and signs of visible cerebral contusions at the impact site were avoided. Secondly, this microdialysis technique successfully replicated the previously demonstrated the acute and short-lived extracellular glutamate release taking place within the first 10 min following trauma induction ${ }^{14,16}$. Moreover, keeping the probe inserted throughout the whole procedure significantly reduces the likelihood of inducing damage to the mTBI-sensitive blood-brain barrier linked to repeated microdialysis probe insertion ${ }^{34}$.

Future applications or directions of the method. 
Given the easy-to-use aspects of the Wayne State University weight-drop procedure and the acute extracellular neurotransmitter level changes measured by microdialysis, our rat model combining microdialysis and concussion provides researchers with a reliable tool to faithfully reproduces the biomechanics of human craniocerebral trauma and longitudinally characterize the molecular effects of concussions. Our rat model could also be used in a wide variety of therapeutical studies as it offers a valuable opportunity to study the mechanism and efficacy of pharmacologic agents in vivo, continuously and without having to sacrifice the animal. Furthermore, the availability of a rat model such as the one presented here could greatly facilitate the better understanding of the relationship between the neurotransmitter imbalances and the behavioral consequences of concussions.

\section{Disclosures}

No competing financial interests exist.

\section{Acknowledgments}

We are grateful to Louis Chiocchio for animal care and maintenance, Morgane Regniez for assistance with the intracardiac perfusion procedure, and David Castonguay for assistance with the cryostat. This work was supported by the Caroline Durand Foundation Chair in acute traumatology of the Universite de Montreal awarded to LDB.

\section{References}

1. Cassidy, J. D. et al. Incidence, risk factors and prevention of mild traumatic brain injury: results of the WHO Collaborating Centre Task Force on Mild Traumatic Brain Injury. Journal of Rehabilitation Medecine. (43 Suppl), 28-60 (2004).

2. McCrory, P. et al. What is the definition of sports-related concussion: a systematic review. British Journal of Sports Medecine. 51 (11), 877-887 (2017).

3. McCrory, P. et al. 5th International Conference on Concussion in Sport (Berlin). British Journal of Sports Medecine. 51 (11), 837 (2017).

4. Cernak, I. Animal models of head trauma. NeuroRx. 2 (3), 410-422 (2005).

5. Davis, A. E. Mechanisms of traumatic brain injury: biomechanical, structural and cellular considerations. Critical Care Nursing Quarterly. 23 (3), 1-13 (2000).

6. Gaetz, M. The neurophysiology of brain injury. Clinical neurophysiology : official journal of the International Federation of Clinical Neurophysiology. 115 (1), 4-18 (2004).

7. Giza, C. C., \& Hovda, D. A. The new neurometabolic cascade of concussion. Neurosurgery. 75 Suppl 4 S24-33 (2014).

8. Guerriero, R. M., Giza, C. C., \& Rotenberg, A. Glutamate and GABA imbalance following traumatic brain injury. Current neurology and neuroscience reports. 15 (5), 27 (2015).

9. Meldrum, B. S. Glutamate as a neurotransmitter in the brain: review of physiology and pathology. Journal of Nutrition. 130 (4S Suppl), 1007S-1015S (2000).

10. Morris, R. G., Garrud, P., Rawlins, J. N., \& O'Keefe, J. Place navigation impaired in rats with hippocampal lesions. Nature. 297 (5868), 681-683 (1982).

11. Olton, D. S., \& Papas, B. C. Spatial memory and hippocampal function. Neuropsychologia. 17 (6), 669-682 (1979).

12. Ray, S. K., Dixon, C. E., \& Banik, N. L. Molecular mechanisms in the pathogenesis of traumatic brain injury. Histology and histopathology. 17 (4), 1137-1152 (2002).

13. Reger, M. L. et al. Concussive brain injury enhances fear learning and excitatory processes in the amygdala. Biological Psychiatry. 71 (4), 335-343 (2012).

14. Faden, A. I., Demediuk, P., Panter, S. S., \& Vink, R. The role of excitatory amino acids and NMDA receptors in traumatic brain injury. Science. 244 (4906), 798-800 (1989).

15. Folkersma, H. et al. Increased cerebral (R)-[(11)C]PK11195 uptake and glutamate release in a rat model of traumatic brain injury: a longitudinal pilot study. Journal of neuroinflammation. 867 (2011).

16. Katayama, Y., Becker, D. P., Tamura, T., \& Hovda, D. A. Massive increases in extracellular potassium and the indiscriminate release of glutamate following concussive brain injury. Journal of neurosurgery. 73 (6), 889-900 (1990).

17. Nilsson, P., Hillered, L., Ponten, U., \& Ungerstedt, U. Changes in cortical extracellular levels of energy-related metabolites and amino acids following concussive brain injury in rats. Journal of cerebral blood flow and metabolism : official journal of the International Society of Cerebral Blood Flow and Metabolism. 10 (5), 631-637 (1990).

18. Kane, M. J. et al. A mouse model of human repetitive mild traumatic brain injury. Journal of neuroscience methods. 203 (1), 41-49 (2012).

19. Dewitt, D. S., Perez-Polo, R., Hulsebosch, C. E., Dash, P. K., \& Robertson, C. S. Challenges in the development of rodent models of mild traumatic brain injury. Journal of Neurotrauma. 30 (9), 688-701 (2013).

20. Eisenberg, M. A., Andrea, J., Meehan, W., \& Mannix, R. Time interval between concussions and symptom duration. Pediatrics. 132 (1), 8-17 (2013).

21. Guskiewicz, K. M. et al. Cumulative effects associated with recurrent concussion in collegiate football players: the NCAA Concussion Study. JAMA. 290 (19), 2549-2555 (2003).

22. Luo, J. et al. Long-term cognitive impairments and pathological alterations in a mouse model of repetitive mild traumatic brain injury. Frontiers in neurology. 512 (2014).

23. Meehan, W. P., 3rd, Zhang, J., Mannix, R., \& Whalen, M. J. Increasing recovery time between injuries improves cognitive outcome after repetitive mild concussive brain injuries in mice. Neurosurgery. 71 (4), 885-891 (2012).

24. Prins, M. L., Hales, A., Reger, M., Giza, C. C., \& Hovda, D. A. Repeat traumatic brain injury in the juvenile rat is associated with increased axonal injury and cognitive impairments. Developmental neuroscience. 32 (5-6), 510-518 (2010).

25. Schwetye, K. E. et al. Traumatic brain injury reduces soluble extracellular amyloid-beta in mice: a methodologically novel combined microdialysis-controlled cortical impact study. Neurobiology of disease. 40 (3), 555-564 (2010). 
26. Shitaka, Y. et al. Repetitive closed-skull traumatic brain injury in mice causes persistent multifocal axonal injury and microglial reactivity. Journal of neuropathology and experimental neurology. 70 (7), 551-567 (2011).

27. Willie, J. T. et al. Controlled cortical impact traumatic brain injury acutely disrupts wakefulness and extracellular orexin dynamics as determined by intracerebral microdialysis in mice. Journal of neurotrauma. 29 (10), 1908-1921 (2012).

28. Bolton, A. N., \& Saatman, K. E. Regional neurodegeneration and gliosis are amplified by mild traumatic brain injury repeated at 24 -hour intervals. Journal of neuropathology and experimental neurology. 73 (10), 933-947 (2014).

29. Blaylock, R. L., \& Maroon, J. Immunoexcitotoxicity as a central mechanism in chronic traumatic encephalopathy-A unifying hypothesis. Surgical neurology international. 2107 (2011).

30. McCrory, P., Davis, G., \& Makdissi, M. Second impact syndrome or cerebral swelling after sporting head injury. Current Sports Medecine Reports. 11 (1), 21-23 (2012).

31. Fujita, M., Wei, E. P., \& Povlishock, J. T. Intensity- and interval-specific repetitive traumatic brain injury can evoke both axonal and microvascular damage. Journal of Neurotrauma. 29 (12), 2172-2180 (2012).

32. Paxinos, G., \& Watson, C. The Rat Brain in Stereotaxic Coordinates. 4th edn, Academic Press (1998).

33. Bales, J. W., Wagner, A. K., Kline, A. E., \& Dixon, C. E. Persistent cognitive dysfunction after traumatic brain injury: A dopamine hypothesis. Neuroscience \& Biobehavioral Reviews. 33 (7), 981-1003 (2009).

34. Sumbria, R. K., Klein, J., \& Bickel, U. Acute depression of energy metabolism after microdialysis probe implantation is distinct from ischemiainduced changes in mouse brain. Neurochemical Research. 36 (1), 109-116 (2011). 